\title{
Tendencias tecnológicas en el sector biotecnológico: análisis de patentes en México y Estados Unidos ${ }^{1}$
}

\author{
Technological Trends in the Biotechnology Sector: \\ Patent Analysis in Mexico and the United States
}

\begin{abstract}
Mario Alberto Morales Sánchez, ${ }^{2}$ Marcela Amaro Rosales ${ }^{3}$ y Federico Andrés Stezano Pérez ${ }^{4}$
\end{abstract}
\section{RESUMEN}

Esta investigación tiene como objetivo comparar tendencias tecnológicas de patentamiento en biotecnología en Estados Unidos y México, con la finalidad de identificar patrones convergentes entre ambos países durante el periodo 2009-2014, para determinar si las tecnologías que se patentan en México se equiparan con las que se patentan en el país líder en el desarrollo biotecnológico a nivel mundial.

El sector biotecnológico se define con base en la clasificación propuesta por la Organización para la Cooperación y el Desarrollo Económicos (OCDE), a partir del uso de la Clasificación Internacional de Patentes (CIP) y se propone una nueva subdivisión sectorial de la biotecnología.

Los resultados obtenidos muestran que a pesar de que las trayectorias tecnológicas son similares en las empresas que patentan en ambos países, las mexicanas presentan un desempeño pobre, lo cual refleja que en trayectorias tecnológicas globales las ventanas de oportunidad para las empresas nacionales se sitúan en tecnologías pre-paradigmáticas, rango en el que aspiran a desarrollar estrategias imitativas tempranas en nichos no explotados por las empresas transnacionales, pero no se sitúan en la frontera tecnológica mundial.

Palabras clave: desarrollo tecnológico, patentes, biotecnología Clasificación JEL: O30, O34.

\section{Abstract}

The objective of this research is to compare technology trends in biotechnology patenting in the United States and Mexico, in order to identify patterns of convergence between the

\footnotetext{
1 Fecha de recepción: 08/02/2018. Fecha de aprobación: 28/01/2019.

2 Universidad Nacional Autónoma de México, México. Correo electrónico: albertoms@economia.unam.mx. ORCID: 0000-0002-2318-7424.

3 Universidad Nacional Autónoma de México, México. Correo electrónico: marcela.amaro@sociales.unam.mx. ORCID: 0000-0002-1647-8901.

4 Instituto Tecnológico y de Estudios Superiores de Monterrey. México. Correo electrónico: fstezano@gmail.com. ORCID: 0000-0001-5450-6339.
} 
two countries in the 2009-2014 period and determine whether the technologies patented in Mexico are comparable to those that are patented in the country that leads the world in biotechnological development.

Based on the classification proposed by the Organization for Economic Cooperation and Development (OECD), the biotechnology sector definition is based on the use of the International Patent Classification (CIP), and a new sectoral subdivision of biotechnology is proposed.

The results obtained show that although the technological routes are similar in the companies that patent in both countries, Mexican companies display poor performance, which reflects that, in global technological development, the windows of opportunity for national companies are situated in pre-paradigmatic technologies, where they aspire to develop early imitative strategies in niches not exploited by transnational corporations but not on the frontier of world technology.

Key words: Technological development, patents, biotechnology JEL Classification: O30, O34. 


\section{INTRODUCCIÓN}

Esta investigación tiene como objetivo comparar tendencias tecnológicas de patentamiento en biotecnología entre Estados Unidos y México, con la finalidad de identificar patrones convergentes entre ambos países durante el periodo 20092014, para determinar si las tecnologías que se patentan en México se equiparan con las que se patentan en el país líder en el desarrollo biotecnológico a nivel mundial.

Para el caso exclusivo de empresas mexicanas se realiza una comparación análoga, que permite identificar ciertas ventanas de oportunidad para las empresas locales. La pregunta de investigación indaga sobre la existencia de procesos de convergencia o divergencia entre las tecnologías que se patentan en México y las que se patentan en Estados Unidos, y busca determinar qué tan cerca o lejos se encuentran las empresas nacionales de estas tendencias generales. Como hipótesis se plantea que las empresas nacionales tienen dificultades estructurales inherentes para insertarse en los mercados mundiales de biotecnología debido a las escasas capacidades tecnológicas y de innovación que poseen y a la estructura del mercado biotecnológico.

Las empresas biotecnológicas líderes a nivel mundial poseen una capacidad de concentración de mercado que les permite establecer fuertes barreras de entrada (tecnológicas, cognitivas y de mercado) a nuevos competidores, como sería el caso de empresas mexicanas, además de que éstas tienden a poseer mayores limitaciones en sus capacidades científicas, de absorción tecnológica y financieras. Pese a esto, las empresas locales han desarrollado algunas estrategias de inserción que se analizarán en este trabajo.

Distintos conceptos han buscado definir la naturaleza del cambio tecnológico: regímenes tecnológicos, trayectorias, paradigmas, diseños dominantes (Cimoli y Dosi, 1995: 245). En particular, el concepto de trayectorias tecnológicas busca explicar patrones dinámicos del cambio tecnológico que distinguen a los sectores. Ante las distintas fases de una trayectoria tecnológica es posible encontrar distintos regímenes de innovación y condiciones de entrada a nuevos agentes en el sector (Klepper, 1997; Pérez, 2001). Las empresas de países en desarrollo afrontarán distintos contextos de competencia dependiendo del grado de consolidación de sus tecnologías y de la fase de la trayectoria del paradigma tecnológico de interés.

Comparadas con las empresas líderes en el mercado mundial, las empresas nacionales cuentan con distintas posibilidades para: i) diversificar sus bases cognitivas a partir del desarrollo de conocimientos biotecnológicos en sus Departamentos de Investigación y desarrollo (I+D) y ii) acceder a fuentes externas de conocimiento por la vía de colaboraciones con universidades y centros de investigación. Por tanto, las empresas de los países en vías de desarrollo dependen 
de las estrategias competitivas de las empresas líderes del sector (Gutman y Lavarello, 2014: 17-39).

Este trabajo plantea un análisis comparativo entre las tecnologías que han desarrollado las empresas del sector biotecnológico en México y en Estados Unidos. Lo anterior se plantea a partir de identificar patentes biotecnológicas provenientes de dos bases de datos: una nacional, la del Instituto Mexicano de Propiedad Industrial, en adelante IMPI, y otra internacional, la de la Oficina de Patentes de Estados Unidos, en adelante uspto).

El trabajo se presenta en el siguiente orden. En la primera sección se desarrolla el marco teórico-conceptual, en la segunda se plantea el esquema metodológico que articula el análisis realizado de las patentes biotecnológicas, en la tercera se analiza el contexto general de la biotecnología y en la última sección se presenta el estudio sobre las tendencias de patentamiento. Por último, en las conclusiones se retoman los principales resultados arrojados en la sección precedente.

\section{TRAYECTORIAS TECNOLÓGICAS SECTORIALES, PROCESOS DE INNOVACIÓN Y BIOTECNOLOGÍA}

Dos supuestos centrales son inherentes al concepto de trayectorias tecnológicas: la existencia de procesos de dependencia del sendero (path-dependence) y la existencia de un carácter sectorial de la innovación. Los procesos de acumulación tecnológica se muestran limitados por paradigmas tecnológicos y dependientes de ciertas trayectorias tecnológicas (Castellacci, 2007).

Ante la emergencia y difusión de un nuevo paradigma tecnológico en un sistema económico, las respuestas de las industrias difieren en la medida en que son capaces de explotar las trayectorias tecnológicas imperantes en ese paradigma. Tales diferencias permiten explicar tanto la subida como la caída de diferentes industrias y tecnologías (Nelson y Winter, 1977: 60).

La perspectiva de sistemas sectoriales permite concebir las actividades de los actores determinantes en los procesos sectoriales de la innovación: proveedores, usuarios, clientes y consumidores; universidades y centros públicos de investigación; organizaciones financieras (bancos, mercados de capitales y financiamiento interno); el gobierno y sus políticas públicas de innovación, ciencia y tecnología. Estos actores se encuentran conectados en redes y clústers de innovación, producción y distribución que integran complementariedades en conocimiento, capacidades y especialización (Malerba y Nelson, 2011: 1650-1651). 
El desempeño del sector biotecnológico a nivel nacional (comprendido como cadena de valor o como sistema sectorial de innovación ${ }^{5}$ ) depende de las acciones conjuntas que emprenden los agentes del sector; de la configuración sectorial de las estructuras de mercado (incubadoras, fondos de inversión, capital semilla, capitales de riesgo, mercados de capitales, instrumentos bancarios) y de las dinámicas tecnológicas (el modo en que las tecnologías básicas afectan la naturaleza, fronteras y organización de los sectores, las bases sectoriales de conocimiento y los procesos de aprendizaje). La empresa biotecnológica, como organización, internaliza conocimientos de distintas fuentes del sistema y estructura su actividad innovadora a partir de vinculaciones en los niveles local, regional o global (Malerba, 2004).

El desarrollo y las dinámicas actuales del paradigma biotecnológico han estado marcados por cuatro cambios fundamentales: en su base cognitiva, en el régimen de aprendizaje, en su estructuración institucional y en la aparición de un nuevo tipo de agentes innovadores.

A nivel cognitivo, las biotecnologías modernas se caracterizan por una nueva base cognitiva generada a partir de las llamadas revoluciones del ácido desoxirribonucleico (ADN) y la genética molecular, el advenimiento de la biología molecular y los avances significativos en la fisiología, la farmacología, la enzimología y la biología celular (McKelvey et al., 2004: 89). Estos cambios primero impactaron en el campo de la farmacéutica y luego, a partir de éstos, afectaron también en forma significativa al sector productivo y agrícola.

Adicionalmente, tres profundos cambios institucionales reestructuraron al sector biotecnológico: i) la decisión, en 1980, de la Suprema Corte de Estados Unidos de extender la protección a organismos vivos; ii) el acta Bayh-Dole y iii) la directriz de la Oficina de Patentes y Marcas estadounidense, establecida en 1987, en el sentido de patentar cualquier organismo vivo pluricelular (excepto humanos), en tanto se consideraba que la identificación y clasificación de las propiedades y funciones de un gen eran condición suficiente para reclamar el invento de ese gen (véase Coriat et al., 2003). Así, desde los años ochentas comenzó a prevalecer un modelo global de negocios e innovación en el sector al estilo estadounidense, basado en una fuerte investigación científica pública licenciada a empresas privadas y la creación de nuevas empresas con fuertes derechos de

\footnotetext{
Ambas perspectivas coinciden en centrarse en el mapeo y caracterización de los actores y sus interacciones. No obstante, el énfasis analítico que cada perspectiva privilegia respecto al mapeo de los agentes y sus vínculos difiere significativamente. La visión de sistemas de innovación (a nivel nacional, sectorial e incluso regional) se centra en los procesos y dinámicas de generación y uso del conocimiento, en ocasiones en una etapa particular de una cadena de valor. En cambio, el enfoque de cadenas de valor atiende antes a los procesos de creación de valor, las oportunidades de mercado y los vínculos a lo largo de una cadena (Anandajayasekeram y Gebremedhin 2009).
} 
propiedad intelectuales establecidos y con un alto protagonismo de los inversores de riesgo para financiar nuevas empresas (Sztulwark, 2012).

Los cambios referidos modificaron la estructura organizacional de las actividades de innovación y propiciaron el rediseño de los patrones de división del trabajo, las estructuras de incentivos y los mecanismos de selección de los agentes. La manifestación más trascendente en el sector tiene que ver con la aparición de un nuevo tipo de agentes: las empresas biotecnológicas especializadas o nuevas empresas biotecnológicas que, junto con las grandes empresas, integradas verticalmente, constituyen los dos tipos de actores empresariales dominantes del sector.

En primer lugar, las empresas biotecnológicas especializadas se concentran en el desarrollo del potencial comercial de adelantos en Ciencia y tecnología (C y $\mathrm{T}$ ) realizados por investigadores de universidades y hospitales, para trasladar el conocimiento generado al sector científico público y transformarlo en técnicas y productos comercialmente útiles (McKelvey et al., 2004: 92). Muchas de estas empresas pasan años e incluso décadas en transitar de un descubrimiento científico a un producto comercializable, pues suelen carecer de los recursos necesarios para fabricar, distribuir y comercializar sus invenciones. Su modelo de negocios depende, por tanto, de obtener financiamiento del capital de riesgo, de la venta de licencias de conocimiento especializado a grandes firmas o del desarrollo de investigación para las mismas, por la vía de contratos o alianzas conjuntas.

Para estas empresas es difícil llegar al mercado por sí mismas ya que deben cumplir dos requisitos complejos en un sector con fuertes barreras de entrada y pocos grandes jugadores dominantes: i) tener los recursos para cubrir costos de regulación e $\mathrm{I}+\mathrm{D}$ y ii) contar con una infraestructura de marketing (Wield et al., 2010).

En segundo lugar, en el modelo dominante de la gran empresa, verticalmente integrada, ésta se involucra en la mayoría de las actividades para desarrollar y comercializar un nuevo producto o proceso biotecnológico, incluyendo I+D, producción, distribución y marketing. Las empresas que reciben ingresos de la venta de productos biotecnológicos y del desarrollo de sus propios productos proporcionan, además, un mercado para los descubrimientos de las empresas biotecnológicas especializadas (OECD, 2009: 164-167). Una característica común de estas grandes empresas ha estado determinada por la búsqueda de la ampliación de su base de conocimiento (esfuerzos por aumentar sus capacidades de investigación y absorción) y por una intensa profundización de sus vínculos con la comunidad científica financiada públicamente, con base en una estrategia centrada en la consolidación como actores activos de la arena científica y no observadores pasivos o simples usuarios (McKelvey et al., 2004: 94-95).

Además de los aspectos ya anotados, un elemento crucial que permite el análisis de los sistemas sectoriales de innovación y de sus potencialidades futuras 
es el aspecto tecnológico; es decir, qué tipo de tecnologías lo constituyen. Por tal motivo se intenta comparar las tecnologías que conforman el sistema biotecnológico mexicano con su contraparte más dinámica a nivel internacional: el sector biotecnológico estadounidense. Para lograrlo se utilizan las patentes como una variable cualitativa aproximada de las capacidades tecnológicas existentes en un sistema determinado.

Comprender en el sector biotecnológico la coordinación depende de los arreglos institucionales que articulan las relaciones entre los agentes. En este sentido, el acceso diferencial a los derechos tiene una importancia central. Si bien el grado de articulación entre la ciencia y la industria está condicionado por la existencia de complementariedades tecnológicas, se encuentra mediado por derechos y normas que regulan el mercado.

En un esquema dominado por arreglos de mercado, lo que posibilita que un conocimiento se transforme en una mercancía es la existencia de un derecho de propiedad intelectual y un derecho a comercializar.

En el sector biotecnológico, el liderazgo de las empresas transnacionales se basa en mantener un rol clave en la definición de este conjunto de derechos, de allí la importancia creciente que adquiere el marco regulatorio en propiedad intelectual como factor crítico que puede condicionar las estrategias de las empresas.

En el contexto institucional del sector, las empresas usan las patentes como mecanismos de apropiación de la innovación y como activos estratégicos para crear y mantener posiciones dominantes. Las transacciones en los mercados de tecnología (licencias de patentes) son un componente de los nuevos mercados de conocimiento. Además, persiste un antiguo mercado (el de la ciencia) en el que los jugadores (universidades) obtienen patentes derivadas de la investigación básica, por lo que los agentes universitarios protegen $\mathrm{I}+\mathrm{D}$ básica antes no patentable.

Asimismo, se crean mercados adicionales en los que las patentes son un activo estratégico para redefinir posiciones jerárquicas de las empresas y aumentar su poder de negociación. Las patentes se vuelven activos líquidos, intercambiables entre empresas, que permiten resolver disputas legales y facilitar acuerdos de licencias cruzadas. Las expectativas sobre el valor de una patente y la posibilidad de combinar patentes de bajo valor en carteras de patentes animan a las empresas a desarrollar estrategias agresivas. Emerge entonces otro mercado de conocimiento (derivado) en el que las firmas solicitan patentes con el fin explícito de no usarlas en transacciones con otras empresas.

En este mercado, las patentes tienen un valor en sí basado en expectativas de futuro: por la potencial importancia de la innovación patentada para futuras innovaciones, para bloquear la entrada de nuevos competidores o para aumentar 
el valor de la firma al indicar capacidades tecnológicas potenciales (Cimoli y Primi, 2008: 20-21).

Una patente es un derecho de propiedad otorgado por el gobierno de un país. Las patentes garantizan que el conocimiento de los inventos se encuentre accesible a todos, pero limita su uso comercial a quienes son dueños de las mismas. Las patentes también garantizan un derecho de monopolio temporal de la invención; son, pues, un título de propiedad que tiene validez en el tiempo y en el espacio (Foray, 2004).

La Organización Mundial de Propiedad Intelectual (OMPI) señala tres aspectos problemáticos que han sido objeto de debate en torno a las patentes biotecnológicas. El primero se vincula con las normas jurídicas referentes al alcance de la protección por patente de las invenciones en biotecnología. El segundo se relaciona con la concesión de licencias y la explotación de las patentes en biotecnología (Carullo y Vismara, 2009) y, por último, el tercero hace referencia al desajuste entre diversos sistemas de propiedad intelectual en naciones distintas, o a las contradicciones que pueden prevalecer en un mismo sistema de propiedad intelectual.

Las patentes no reflejan todo el conocimiento tecnológico que se desarrolla en el sector, pero resultan un adecuado indicador proxy para identificar capacidades tecnológicas, sobre todo en sectores de vanguardia tecnológica, intensivos en conocimiento y relacionados de modo directo con las actividades científicas, como la biotecnología (Jaffe, 1986). Por lo anterior, este trabajo utiliza el indicador de patentes como variable que refleja tendencias tecnológicas del sector, a partir del supuesto de que las tecnologías en las que más patentes se registran marcan el ritmo de la investigación y determinan la importancia de ciertas áreas de conocimiento, además de representar una aproximación de las capacidades tecnológicas de un sector y dar cuenta del tipo de trayectorias tecnológicas. Dado que se conocen las limitantes que presentan las patentes para el análisis aquí propuesto, se ha considerado también retomar los resultados de diversos estudios de caso que complementan la explicación sobre la dinámica de las trayectorias tecnológicas y las oportunidades para las empresas mexicanas en el contexto global.

\section{METODOLOGÍA}

Un gran reto analítico y metodológico vinculado al manejo de bases de datos relacionadas con patentes es la definición del sector que se desea estudiar en los 
términos de la Clasificación Internacional de Patentes (CIP). ${ }^{6}$ Es decir, el principal problema es determinar cuáles son los componentes de la CIP que definen la actividad inventiva en un sector específico. Esta dificultad es mayor en los sectores económicos emergentes que combinan de manera transversal diferentes disciplinas y técnicas científicas, como es el caso de la biotecnología.

En el sistema de clasificación propuesto por la OMPI cada tecnología se encuentra codificada con un símbolo que combina caracteres alfanuméricos (denominados en adelante CIPs), a fin de facilitar la búsqueda y el manejo de información. Este es, además, un código universal utilizado por las oficinas de patentes más importantes en el mundo, por lo que permite realizar análisis comparativos como el que aquí se propone. El trabajo retoma el método y la clasificación propuesta por la OCDE para definir al sector biotecnológico (OCDE, 2000). La clasificación comprende un conjunto de CIPs en los que se concentra la actividad inventiva del sector biotecnológico. Cada uno representa una tecnología específica con un grado de generalidad que varía en función de la codificación.

Tomando en cuenta la clasificación de la OCDE (2000) se procedió a conformar una base de datos del sector biotecnológico en México con información publicada por el IMPI. ${ }^{7}$ Dicha base contiene todas las patentes otorgadas en México que coinciden con cada uno de los CIPs que conforman el sector, la cual comprende 7270 registros en el periodo 2009-2014.

Esta información permite identificar las tecnologías que constituyen el sistema sectorial de innovación mexicano, medidas a partir de la dinámica de patentamiento. Los datos señalados facilitan comparar dicha dinámica con la de Estados Unidos, tomado como referencia debido a que es el productor más importante de biotecnología a nivel mundial. La información para determinar las trayectorias tecnológicas en Estados Unidos provienen de la oficina de patentes de ese país. ${ }^{8}$ La búsqueda de las patentes otorgadas por USPTO en Estados Unidos se realizó tomando en cuenta la clasificación de la OCDE ya referida. Cabe señalar que no se utiliza una misma base de datos para el análisis comparativo porque el objetivo no es comparar las tecnologías que patentan empresas mexicanas con respecto a otras de otros países en un mismo sistema sectorial de innovación. Lo que aquí se pretende comparar es la base tecnológica del sistema biotecnológico mexicano con respecto a su contraparte estadounidense, y dentro de este contexto qué patentan las empresas mexicanas en ambos contextos

\footnotetext{
${ }^{6}$ Clasificación propuesta por la Organización Mundial de la Propiedad Intelectual (OMPI). Puede consultarse en http://www.wipo.int/portal/en/index.html

7 En: http://www.impi.gob.mx/

8 En: http://www.uspto.gov/
} 
institucionales. Por lo mismo, es necesario comparar los registros de dos bases de datos diferentes.

Una vez definido el sector de la biotecnología en términos de los CIPs, se procedió a definir subsectores tecnológicos. Los CIPs propuestos por la OCDE para definir al sector, fueron reagrupados en tres grandes subsectores tecnológicos: agrícola, farmacéutico y tecnologías de aplicación general. Este último subsector hace referencia a aquellas tecnologías multipropósito que pueden ser utilizadas en distintas áreas tecnológicas y científicas.

Cabe destacar que esta propuesta de subdivisión sectorial es el aporte metodológico central de este trabajo, y tiene como objetivo analizar las diferencias sectoriales dentro de la biotecnología. En otras palabras, se plantea la originalidad metodológica de la propuesta de subdivisión, ya que para realizarla se contó con la ayuda de expertos en biotecnología, provenientes del IBT, quienes junto con los autores de este trabajo revisaron cada una de las tecnologías que componen cada una de las CIPs antes expuestas, a fin de determinar qué tipo de biotecnología constituyen.

Finalmente, la base de datos construida a partir de la información del IMPI y del USPTO se utilizó también para realizar una aproximación analítica entre desarrollo tecnológico y desarrollo científico. En dicha base se ubicaron las patentes cuyo titular tiene nacionalidad mexicana. El titular puede ser una empresa, un centro de investigación, una universidad u otra entidad académica, e incluso un individuo o grupo de individuos. En el caso de este sector, un amplio porcentaje de patentes en México pertenece a centros de investigación y universidades.

Las bases de datos se construyeron con información proveniente de dos fuentes: la Oficina de Propiedad Industrial mexicana (IMPI) y la oficina de patentes de Estados Unidos (USPTO). En ambos casos se obtuvieron todas las patentes concedidas en biotecnología durante el periodo de estudio. La busca se realizó a partir de la clasificación referida de la OECD, en la que se define el sector biotecnológico en términos de CIPs (códigos internacionales de clasificación de patentes). Dicha clasificación se compone por un conjunto de CIPs que, de acuerdo con los expertos de la OECD, comprenden todas las tecnologías registradas en el catálogo de la Organización Mundial de la Propiedad Intelectual, consideradas como aplicaciones biotecnológicas. La extracción de la información se realizó con motores de búsqueda estándar a partir de la clasificación descrita por la OECD. Se obtuvieron todas las patentes cuya clasificación principal o alguna de sus clasificaciones adicionales coincidieran con alguno de los CIPs considerados por la OECD como aplicaciones biotecnológicas. Se trabajó sólo con las patentes concedidas para poder homologar la información de las distintas fuentes de información, ya que en el caso del IMPI no se contaba con los registros de las patentes solicitadas. Debido a que en Estados Unidos se concede un número de patentes mucho mayor que 
en México, se crearon histogramas de frecuencia relativa con respecto a cada uno de los CIPs para poder comparar las trayectorias tecnológicas en cada país independientemente del número absoluto de patentes.

\section{CONTEXTO BIOTECNOLÓGICO}

Antes de desarrollar al análisis pormenorizado del comportamiento de las CIPs para identificar la convergencia o divergencia de las tecnologías que se patentan en México y Estados Unidos, es necesario contextualizar de manera sintética el entorno de la biotecnología en México y el porqué se eligió a Estados Unidos, país más relevante en el desarrollo de biotecnología a nivel mundial.

Sin duda alguna Estados Unidos es el país más relevante en términos económicos y tecnológicos para el desarrollo, uso y explotación de la biotecnología a nivel mundial. Así lo revelan diversos estudios que destacan que el mercado de este país ha determinado en gran medida el volumen de la industria, el gasto internacional dedicado a la $\mathrm{I}+\mathrm{D}$, el número de empleos asociados y el número de empresas (ICEX, 2018). Sólo en 2017 se registraron 2270 empresas con ingresos de alrededor de los 106900 millones de dólares, con un total de empleo de 308000 trabajadores, además de contar con las empresas líderes del sector (ICEX, 2018).

Por el tipo de requerimientos financieros que tiene la industria biotecnológica, basados en altas inversiones en I+D y en el proceso de precomercialización y comercialización, el mercado se ha ido concentrando y la tendencia a nivel mundial ha sido la siguiente: la tendencia a la concentración ha ido disminuyendo las oportunidades para las empresas de los países en vías de desarrollo, ya que éstas suelen tener muchos problemas para insertarse en trayectorias tecnológicas exitosas, dado que no cuentan con los recursos necesarios para invertir en $\mathrm{I}+\mathrm{D}$ ni la capacidad de soportar los requerimientos regulatorios, lo que se convierte en barreras de entrada, muchas veces infranqueables.

El panorama anterior muestra la importancia del comportamiento tecnológico y de mercado de las empresas estadounidenses, en vista de que tienen la capacidad de modificar la trayectoria, dada la demanda de conocimiento tecnológico con base en lo que proyectan como espacios con mayor posibilidad de obtener una rentabilidad más alta. Sin duda esto impacta en el comportamiento de la biotecnología a nivel mundial, porque se determinan agendas de $\mathrm{I}+\mathrm{D}$, mercados y posibles áreas para el resto de las empresas.

En América Latina ha primado la existencia de pocas empresas biotecnológicas que realizan actividades de biotecnología moderna. En términos generales, el desarrollo de políticas nacionales de desarrollo de empresas biotecnológicas locales ha sido escaso y discontinuo. En este contexto, las empresas que han cen- 
trado su negocio en la I+D y la innovación son escasas y pueden definirse como islas de conocimiento. Esto es, empresas que aunque no operan en red, centran sus ventajas competitivas en la transformación del conocimiento desde la acumulación de competencias endógenas innovativas y emprendedoras. Sin embargo, especialmente en el caso de algunas empresas de software y biotecnología en América Latina, donde se encuentran casos de empresas con capacidades tecnológicamente relevantes, dado su aislamiento no logran obtener sinergias del ambiente ni generar derramas de conocimiento (Robert, Yoguel y Erbes, 2007: 36).

En las últimas dos décadas, en Brasil y Argentina se ha buscado fortalecer el entorno innovador nacional para fortalecer el proceso de generación de capacidades empresariales desde algunas políticas nacionales de desarrollo científico y tecnológico sectorial. En el caso brasileño, desde el año 2000 hubo un marcado crecimiento del sector biotecnológico como consecuencia de una nueva política industrial que dio un rol protagónico al Estado y relanzó la industria nacional. Lo que parece común en ambos casos es la importancia de una estrategia nacional de políticas de CTI sectoriales que respalden el proceso de desarrollo de capacidades tecnológicas empresariales.

En contraste, de acuerdo con Amaro y Morales (2016), el sistema sectorial de innovación para el caso de la biotecnología en México aún no se encuentra consolidado, ya que aunque existen potencialidades importantes en ciertas áreas científicas y tecnológicas, la aún insuficiente dotación de recursos materiales y humanos, principalmente en las empresas, ha limitado de manera drástica un despliegue tecnológico importante. Pese a que en diversos programas de gobierno a nivel federal y estatal se menciona que la biotecnología representa un sector estratégico para el desarrollo del país no existe ninguna política pública específica que incentive su despliegue.

Con respecto a la existencia de empresas biotecnológicas en México, existen algunos estudios y bases de datos que sitúan su número entre 300 y 400 en todo el país (véase Trejo, 2010; OCDE, 2015); sin embargo, al no realizarse una distinción adecuada entre aquellas que desarrollan tecnología y las que sólo son usuarias de productos biotecnológicos, es muy probable que esta cifra esté sobreestimada. De acuerdo con una encuesta realizada por la Red Temática de Convergencia de Conocimiento para Beneficio de la Sociedad, financiada por CONACYT, levantada entre septiembre de 2015 y enero 2016, existían en total 40 empresas biotecnológicas mexicanas que desarrollan proyectos biotecnológicos, lo cual implica excluir a las que sólo comercializan productos biotecnológicos y/o los utilizan como un insumo, sin tener actividades de asimilación tecnológica; así como a las que forman parte de una empresa transnacional y sus proyectos biotecnológicos no se realizan en territorio mexicano. Los resultados de esta encuesta (por pu- 
blicarse en diversos trabajos) muestran que $33 \%$ de dichas empresas desarrollan procesos biotecnológicos generales, $18 \%$ proteínas y moléculas, $15 \%$ biotecnología ambiental y $10 \%$ tecnologías relacionadas con el ADN.

Si se compara el número de empresas biotecnológicas en México, que suponen son entre 300 y 400 (difícil de determinar por la mortandad alta de las mismas), y las empresas que desarrollan biotecnología (denominadas por la OCDE dedicadas a la biotecnología), que según la misma fuente son alrededor de 40 , con las empresas existentes en Estados Unidos, que son más de 11300 en el primer caso y más de 1100 en el segundo, entonces puede entenderse con facilidad que la actividad empresarial mexicana aún es incipiente.

De acuerdo con Morales y Amaro (2017), la actividad conjunta de diversas instituciones de educación superior, principalmente universidades públicas y centros de investigación, ha dinamizado excepcionalmente el campo de la biotecnología en México a nivel académico, (capacidades científicas y tecnológicas). Por desgracia, estas capacidades son poco utilizadas por la industria. Esta falta de vinculación en gran medida se debe a que el marco regulatorio es débil y hasta la fecha no ha servido para incentivar una mayor actividad de los agentes en el sector. Tampoco existen los incentivos de mercado necesarios para la creación de nuevas empresas biotecnológicas, ni para que las existentes se fortalezcan en el mercado interno y compitan en el mercado internacional.

La falta de una articulación institucional entre los distintos agentes que conforman el sector de alguna manera se explica por la ausencia de un plan estratégico diseñado para el fomento de la biotecnología.

\section{ANÁLISIS DE PATENTAMIENTO Y ESTRATEGIAS TECNOLÓGICAS EMPRESARIALES}

Con base en las CIPs referidas previamente y la clasificación propuesta en este trabajo, a continuación se presenta la composición general de patentes de acuerdo a la definición del sector basada en CIPs. ${ }^{9}$

En la USPTO dominan las patentes que pertenecen al subsector definido como tecnologías de aplicación general (gráfica 1). Lo anterior implica que la mayor parte de la tecnología que allí se patenta es útil para diversas áreas industriales. En el caso de México (gráfica 2) dominan las patentes clasificadas en el subsector farmacéutico, seguidas por las del subsector de tecnologías de aplicación general y las relacionadas con el sector agrícola, lo cual da cuenta de

\footnotetext{
9 En términos de número total de patentes biotecnológicas, el número de patentes registradas en México representa el $10 \%$ del total de las registradas en la USPTO.
} 
la mayor importancia que en la estructura productiva nacional cobra el sector farmacéutico como productor de conocimiento tecnológico. Estos datos revelan una primera diferencia en la conformación de ambos mercados tecnológicos.

Gráfica 1. Distribución de patentes

por clasificación USPTO (2009-2014)

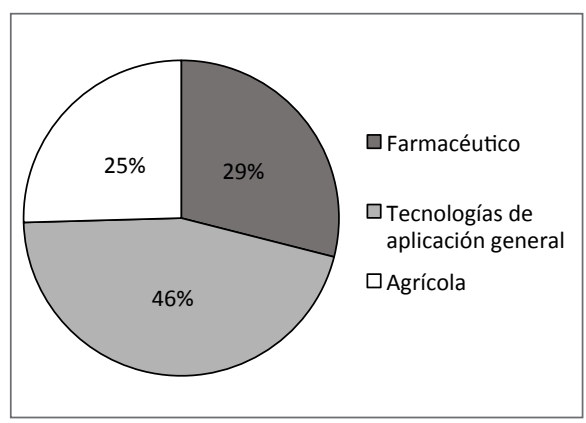

Fuente: elaboración propia.
Gráfica 2. Distribución de patentes por clasificación IMPI (2009-2014)

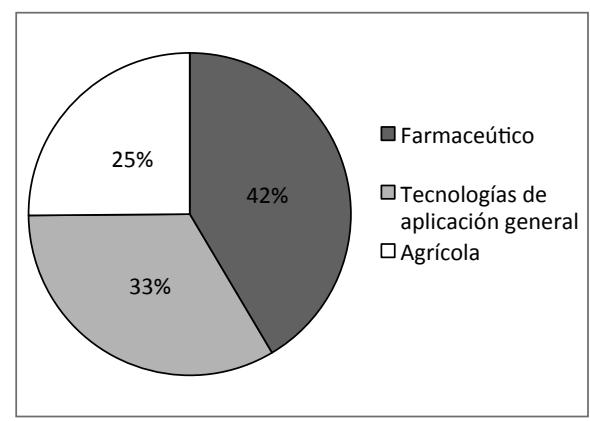

Por su parte, en el cuadro 1 se muestran las principales CIPs en relación con el número de patentes en ambas oficinas, a fin de indicar una aproximación a las diferencias tecnológicas en los dos países. De las patentes registradas en el periodo de 2009 a 2014 en la USPTO (cuadro 1), la principal tecnología se relaciona con el sector agrícola. En segundo y tercer lugares se ubican tecnologías relacionadas con el subsector de tecnologías de aplicación general, mientras que las últimas tres tecnologías conciernen a patentes relacionadas con el sector farmacéutico.

Para el caso del IMPI es interesante destacar que existe coincidencia casi total entre las principales tecnologías en las que más se patenta, aunque con una diferencia en lo relativo al lugar que ocupa cada una. La única excepción es la CIP C12Q que ocupa el séptimo lugar en el IMPI. También se observa que el primer lugar corresponde con la misma tecnología que en el caso de la USPTO, pero en segundo, tercero y cuarto lugares se ubican las CIPs relacionadas con el sector farmacéutico y en los tres últimos lugares se encuentran tecnologías de aplicación general. De esta forma, en términos generales se encuentran ciertas coincidencias entre las principales tecnologías patentadas en México y en Estados Unidos: de las 27 CIPs que comprenden el sector, las seis principales son las mismas en ambos países. 
Esta coincidencia da cuenta también del nivel de maduración de los paradigmas biotecnológicos que se representan en el rol que cumplen distintas biotecnologías en la resolución de problemas en los sectores salud, agrícola e industrial.

Cuadro 1. CIPS que más patentes registran en USPTO e IMPI (2009-2014)

\begin{tabular}{|c|c|c|c|c|c|}
\hline CIP & Tecnología & $\begin{array}{l}\text { Número de } \\
\text { patentes en } \\
\text { USPTO }\end{array}$ & $\begin{array}{l}\text { Lugar que } \\
\text { ocupa en } \\
\text { USPTO }\end{array}$ & $\begin{array}{l}\text { Número de } \\
\text { patentes } \\
\text { en IMPI }\end{array}$ & $\begin{array}{l}\text { Lugar que } \\
\text { ocupa en } \\
\text { IMPI }\end{array}$ \\
\hline $\mathrm{C} 12 \mathrm{~N}$ & $\begin{array}{l}\text { Microorganismos o enzimas; composiciones } \\
\text { que los contienen (biocidas, reguladores de } \\
\text { crecimiento de los vegetales, virus, hongos, } \\
\text { enzimas, fermentación, etc.). }\end{array}$ & 20340 & 1 & 2450 & 1 \\
\hline C12Q & $\begin{array}{l}\text { Proceso de medida, investigación o análisis } \\
\text { en los que intervienen enzimas o } \\
\text { microorganismos }\end{array}$ & 10227 & 2 & 621 & 7 \\
\hline $\mathrm{C} 12 \mathrm{P}$ & $\begin{array}{l}\text { Proceso de fermentación o que utilizan enzimas } \\
\text { para la síntesis de un compuesto químico dado. }\end{array}$ & 9602 & 3 & 788 & 6 \\
\hline A61K38/00 & $\begin{array}{l}\text { Preparaciones medicinales que contienen } \\
\text { péptidos. }\end{array}$ & 9306 & 4 & 1237 & 3 \\
\hline A61K39/00 & $\begin{array}{l}\text { Preparaciones medicinales que contienen } \\
\text { antígenos o anticuerpos. }\end{array}$ & 8243 & 5 & 1418 & 2 \\
\hline C07K14/00 & $\begin{array}{l}\text { Péptidos con más de } 20 \text { aminoácidos; gastrinas; } \\
\text { somatostatinas; etc. }\end{array}$ & 5629 & 6 & 1188 & 4 \\
\hline C07K16/00 & $\begin{array}{l}\text { Inmonoglubinas, anticuerpos mono o } \\
\text { policlonales. }\end{array}$ & 3842 & 9 & 947 & 5 \\
\hline
\end{tabular}

Fuente: elaboración propia.

$\mathrm{Al}$ analizar de manera individual la evolución de cada una de las principales tecnologías, se aprecia que en el caso de la USPTO la CIP C12N mantiene un crecimiento sostenido que supera el de todas las demás. Las trayectorias restantes muestran un ligero crecimiento en el periodo, con excepción de la C12Q que presenta un estancamiento evidente a partir del año 2010 (gráfica 3). Es interesante notar que, a diferencia de lo que ocurre en la USPTO, en el IMPI todas las tecnologías muestran un crecimiento importante hasta el año 2012. Año a partir del cual se registra una caída significativa que llega casi a los mismos niveles que en 2009 en el último año de estudio (gráfica 4). Este comportamiento expresa una baja en el ritmo de patentamiento en los últimos años en México. En consecuencia, la diferencia fundamental radica en que la dinámica creciente del sector biotecnológico se ha mantenido en Estados Unidos, mientras que en México atraviesa un declive importante que no ha podido superarse. 
Gráfica 3. Evolución de las cinco principales CIPS en USPTO (2009-2014)

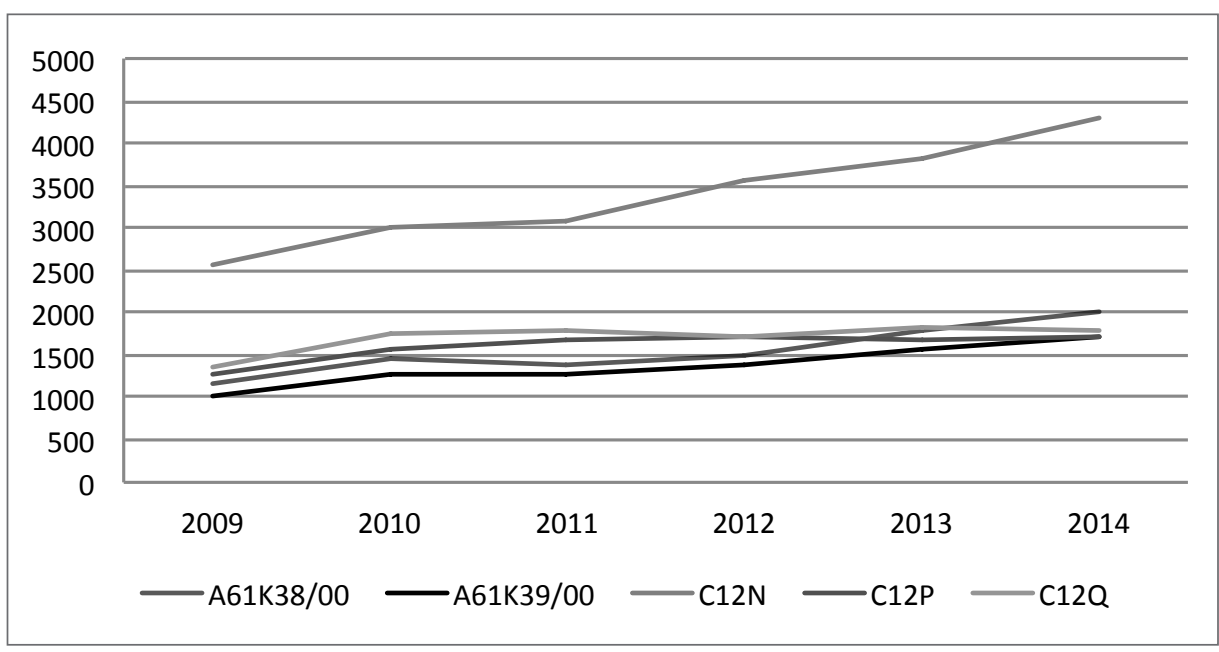

Gráfica 4. Evolución de las cinco principales CIPS en IMPI

(2009-2014)

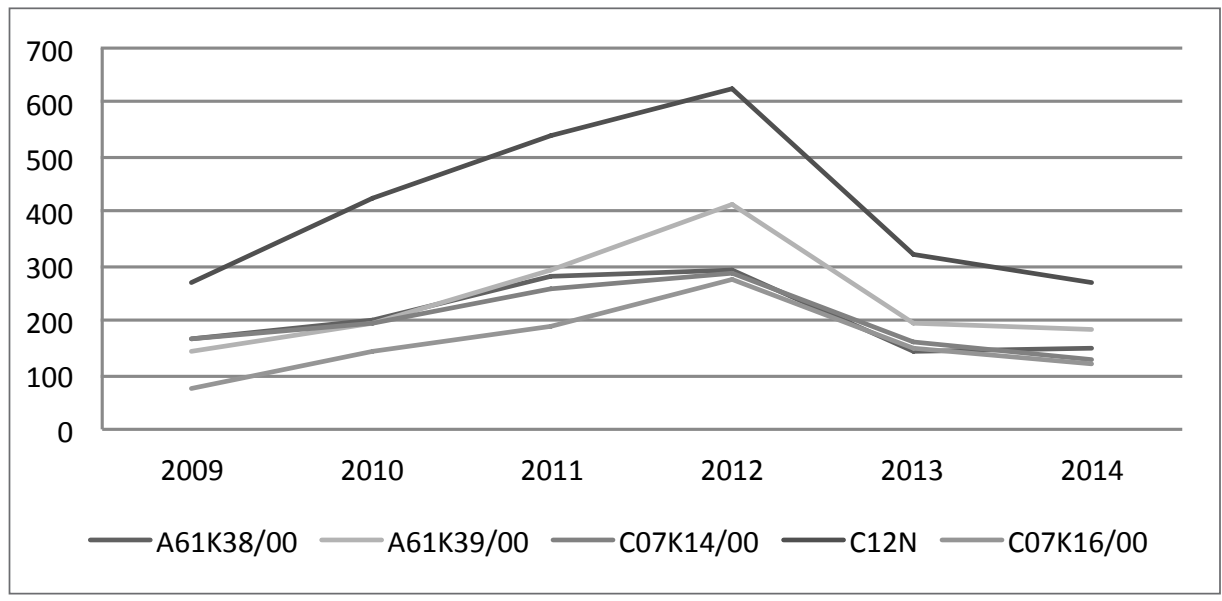

Fuente: elaboración propia. 
Al considerar la titularidad de las patentes, resalta el hecho de que en todo el periodo sólo 50 registran titularidad mexicana en la USPTO. Esto representa una proporción extremadamente baja, de aproximadamente $0.06 \%$ de todas las patentes del sector biotecnológico en Estados Unidos. Esto es indicativo de la baja capacidad de producir conocimiento tecnológico relevante a nivel internacional por parte de los agentes mexicanos. Aún no existen capacidades tecnológicas para competir en el principal mercado biotecnológico.

En la gráfica 5 se observa que del total de patentes registradas con titularidad mexicana en USPTO, $56 \%$ son patentes del sector farmacéutico, seguido por las del sector agrícola y finalmente las tecnologías de aplicación general; es decir que las capacidades tecnológicas más importantes son las del sector farmacéutico.

Pero además, los agentes nacionales muestran carecer también de capacidades tecnológicas suficientes y relevantes para competir en el mercado local. De las 7270 patentes biotecnológicas registradas en el IMPI durante el periodo, sólo $1 \%$ pertenece a titulares mexicanos. Esto implica que la dinámica tecnológica nacional se encuentra determinada fundamentalmente por agentes extranjeros que registran sus principales desarrollos tecnológicos con fines de explotación en el mercado mexicano.

La distribución por áreas de los titulares mexicanos muestra que $43 \%$ se localiza en las denominadas de aplicación general, seguidas por las que pertenecen al subsector agrícolas, con $34 \%$, y finalmente las que pertenecen al subsector farmacéutico que representan el 23 \% (gráfica 6). La principal conclusión respecto a esta distribución es que los agentes nacionales que más patentan son los centros de investigación y las universidades, generalmente relacionados con las tecnologías de aplicación general. Esta conclusión se corrobora con los datos que se muestran más adelante.

Si bien la distribución de las clasificaciones de los titulares mexicanos en ambos países no es coincidente, lo que sí es similar es el bajo nivel de capacidades tecnológicas con que cuentan los agentes nacionales. Esto compromete los beneficios potenciales que este sector puede brindar en términos de desarrollo tecnológico. Se identificó que los titulares mexicanos que patentan en USPTO presentan una muy baja participación en las CIPs más importantes, ya que sólo dos de ellas (C12N y A61K38/00) son patentes de titulares mexicanos. En lo concerniente al IMPI, hay titulares mexicanos en tres de las cinco clasificaciones más importantes. En términos generales, se observa que los titulares mexicanos, a pesar de tener una muy baja participación en cuanto a número de patentes, tienen cierta presencia en las clasificaciones de mayor dinamismo. 
Gráfica 5. Distribución de patentes con titulares mexicanos en USPTO (2009-2014)

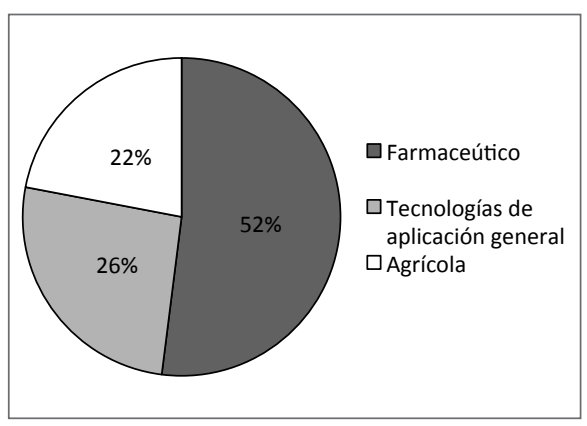

Gráfica 6. Distribución de patentes con titulares mexicanos en IMPI (2009-2014)

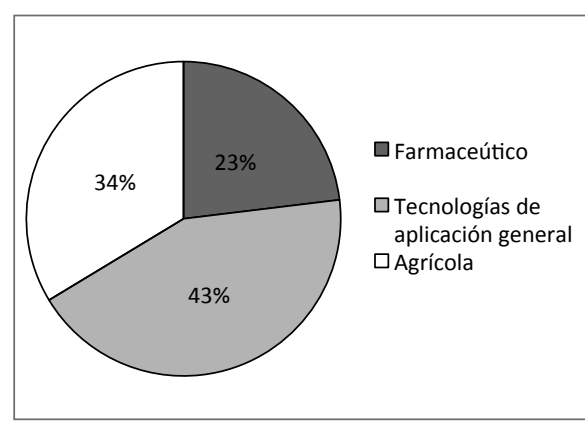

Fuente: elaboración propia.

Los agentes mexicanos que patentan en el IMPI no lo hacen en todos los CIPs que conforman el sector biotecnológico. En ciertas tecnologías no se registra ninguna patente de titular mexicano durante el periodo. De las 27 CIPs que componen el sector, los titulares mexicanos sólo tienen participación en quince. La gráfica 7 muestra las diez principales tecnologías en las que participan los titulares mexicanos en el IMPI. Se puede observar que, con excepción del CIP C12M, que alcanza poco menos del $4 \%$, la participación de titulares mexicanos es menor al $2 \%$ en todos los casos, lo cual expresa la marginalidad de las capacidades tecnológicas en el sector. Si se considera el caso de la USPTO, los titulares mexicanos sólo tienen participación en diez de las 27 CIPs, y su participación porcentual en cada uno de ellos con respecto al total es ínfima. 
Gráfica 7. Porcentaje de titulares mexicanos en relación al total por CIPS (IMPI 2009-2014)

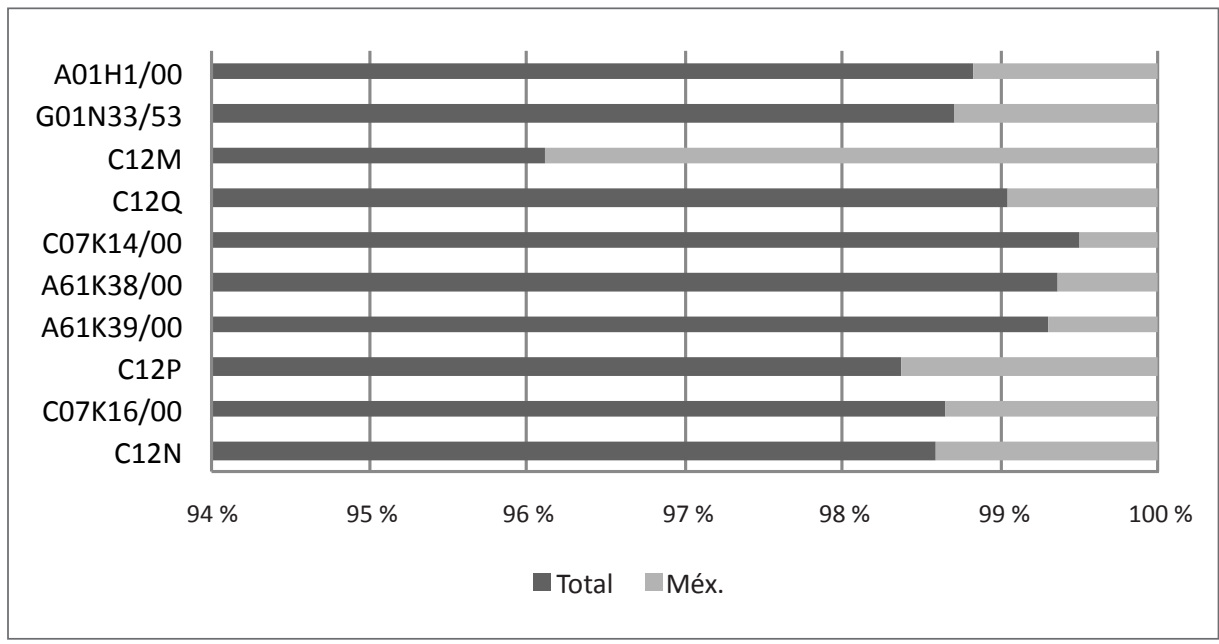

Fuente: elaboración propia.

Finalmente, el otro dato importante que debe ser considerado para el caso mexicano es que del total de patentes de titulares mexicanos registradas en el IMPI en el sector biotecnológico casi el 72 \% corresponde a instituciones de educación superior (IES) y a centros públicos de investigación (CPIs), mientras que sólo el $20 \%$ corresponde a empresas y el $8 \%$ a individuos sin referencia institucional. Esto implica que la generación de conocimiento tecnológico en el sector todavía se encuentra ligado a las actividades de investigación y ha tenido poca repercusión en el sistema productivo nacional.

Más aún, a juzgar por los resultados obtenidos en este trabajo, puede establecerse que existe un rezago muy grande en el sector empresarial en cuanto al desarrollo de capacidades de innovación reflejadas en patentes. Como se muestra en el cuadro 2, en seis años el IMPI sólo ha registrado 18 patentes propiedad de empresas biotecnológicas mexicanas, concentradas en 12 empresas nacionales. Al realizar el mismo análisis para el caso de los registros en la USPTO el problema del rezago se recrudece: existen 22 patentes de empresas biotecnológicas mexicanas registradas en Estados Unidos, que se concentran en seis empresas, de las cuales una, Laboratorios Silanes, posee 10 de las 22 patentes registradas en aquel país. 
Cuadro 2. Empresas mexicanas en el sector biotecnológico con patentes en IMPI y en USPTO (2009-2014)

\begin{tabular}{lllc}
\multicolumn{1}{c}{ Empresa } & $\begin{array}{c}\text { Número de } \\
\text { patentes en IMPI }\end{array}$ & Empresa & $\begin{array}{c}\text { Número de } \\
\text { patentes en USPTO }\end{array}$ \\
\hline TGT Laboratories & 4 & Laboratorios Silanes & 10 \\
\hline Prolec-GE Industrias & 1 & $\begin{array}{l}\text { Boehringer Ingelheim } \\
\text { Vetmédica }\end{array}$ & 3 \\
\hline Laboratorios Silanes & 2 & Instituto Bioclon & 3 \\
\hline METCo & 2 & TGT Laboratories & 1 \\
\hline Boehringer Ingelheim Vetmédica & 1 & Cosmocel & 1 \\
\hline Laboratorio AVI-Mex & 1 & IASA & \\
\hline Empacadora San Marcos & 1 & & \\
\hline Impulsora Nacional de Innovación & 1 & & \\
Tecnológica & 1 & & \\
\hline Cryoinfra & 1 & & \\
\hline Sigma Alimentos & 1 & & \\
\hline Asepro Ecología & 2 & & \\
\hline Alternativas Bioenergéticas & & & \\
\hline
\end{tabular}

Fuente: Amaro y Morales (2016).

Lo anterior parece demostrar que el patentamiento no es una estrategia muy difundida en las empresas biotecnológicas mexicanas, lo cual contrasta fuertemente con las tendencias mundiales referidas en los apartados anteriores. De acuerdo con Morales y Villavicencio (2015: 156-158), 89 \% de las patentes registradas en México por empresas extranjeras en el sector de la biotecnología farmacéutica cuenta con un registro internacional previo, el cual, en promedio, se obtuvo 5.6 años antes en algún otro país. Es decir, que las empresas patentan en México productos que desarrollaron años atrás en algún otro lugar, por lo que el registro de la patente en el mercado local obedece más a una estrategia comercial que al desarrollo de capacidades de innovación. Esta situación distorsiona el mercado local porque fomenta una competencia oligopólica basada en la comercialización de productos, más no en el desarrollo innovador de los mismo y deja poco espacio para que las empresas locales desarrollen procesos de innovación. En estas condiciones no puede esperarse un desarrollo adecuado del sistema sectorial de innovación en biotecnología.

$\mathrm{El}$ análisis de patentes presentado permite identificar, grosso modo, ciertas tendencias de desarrollo tecnológico de las empresas en general que patentan en biotecnología en México y Estados Unidos, y de las empresas mexicanas en particular. 
A fin de atender otro de los objetivos de esta investigación relacionado con la identificación de ventanas de oportunidad para las empresas mexicanas, se ha considerado pertinente retomar los resultados de diversos estudios de caso realizados en las empresas de biotecnología en México, ya que esto pude contribuir a la identificación de estrategias exitosas y de oportunidades potenciales para más empresas nacionales en el sector.

En el cuadro 3 se sintetizan algunos ejemplos de estudios de empresas mexicanas en biotecnología, en los que destacan diversas estrategias que les han permitido mantenerse, diversificarse y/o escalar posiciones en el mercado. Se puede observar que, inicialmente, la gran mayoría de ellas ha implementado estrategias en el desarrollo de productos innovadores con impacto regional o local, ubicándose en ciertos nichos de mercado, por ejemplo el de enfermedades (humanas y animales no humanos), que no son de interés para los grandes laboratorios o bien han aprovechado el vencimiento de patentes para el desarrollo de biosimilares. Lo anterior no quiere decir que éstas sean las únicas estrategias posibles para las empresas biotecnológicas mexicanas, pero se reconoce como un patrón generalizado de aquellas que han logrado posicionarse con éxito. Se observa que no comienzan desarrollando tecnologías de frontera, sino tecnologías relevantes a nivel regional o local, lo cual no es en sí mismo negativo, pero plantea un camino distinto para aprovechar el desarrollo de la biotecnología moderna. Esto puede ser parte de una estrategia de largo plazo que en el futuro les permita insertarse en trayectorias tecnológicas de frontera, una vez que se hayan superado las barreras a la entrada.

Cuadro 3. Estrategias tecnológicas de empresas seleccionadas en biotecnología en México

\begin{tabular}{lll}
\hline \multicolumn{1}{c}{ Empresa } & \multicolumn{1}{c}{ Tecnología } & \multicolumn{1}{c}{ Estrategia } \\
\hline Laboratorios Bioclon & $\begin{array}{l}\text { Farmacéutica- } \\
\text { humana }\end{array}$ & $\begin{array}{l}\text {-Desarrollo de productos innovadores de patente en } \\
\text { mercados nicho de salud. }\end{array}$ \\
\hline Ingenio La Gloria & $\begin{array}{l}\text { Farmacéutica- } \\
\text { humana }\end{array}$ & $\begin{array}{l}\text {-Desarrollo de productos innovadores de patente y } \\
\text { biosimilares. }\end{array}$ \\
\hline Investigación Aplicada & Farmacéutica- animal & $\begin{array}{l}\text {-Desarrollo de productos innovadores para } \\
\text { emandas regionales y locales. }\end{array}$ \\
\hline Nekutli & Alimentos & $\begin{array}{l}\text {-Desarrollo de productos innovadores para } \\
\text { demandas regionales y locales. }\end{array}$ \\
\hline StelaGenomics & Agrícola & $\begin{array}{l}\text {-Desarrollo de semillas innovadoras (reducen el uso de } \\
\text { fosfato y fosfito) sustitutos nacionales e internacionales. }\end{array}$ \\
\hline Fungifree Ab & Agrícola & $\begin{array}{l}\text {-Desarrollo de productos innovadores sustitutos } \\
\text { locales y regionales. }\end{array}$ \\
\hline BioSolutions & Medio ambiente & $\begin{array}{l}\text {-Desarrollo de productos innovadores sustitutos. } \\
\text { Nopalimex }\end{array}$ \\
\hline
\end{tabular}

Fuente: elaboración propia con base en Amaro et al. (2009); Villavicencio et al. (2014); Stezano (2017); de Gortari et al. (2017); Amaro y Morales (2017); Villavicencio y Rivera (2017) y Carrillo y Avendaño (2017). 
En el cuadro anterior se sintetiza parte de los importantes hallazgos de algunos estudios realizados previamente. Vale la pena destacar que todos los casos cuentan con importantes relaciones de vinculación con instituciones de educación superior (IES), lo que les ha permitido desarrollar capacidades tecnológicas y superar importantes barreras a la entrada, como es la inversión inicial en I+D, que suele ser excesivamente alta. Sin embargo, este tipo de estrategias no acostumbran ser suficientes para que las empresas del sector sean competitivas a nivel mundial. Se requieren otros tipos de estrategias de inversión, más agresivas y dirigidas a los mercados internacionales de inversión de riesgo, poco desarrolladas en países como México. Para lograrlo son necesarios al menos dos tipos de capacidades que pocas empresas mexicanas han sido capaces de desarrollar: la capacidad de presentar un producto o un prototipo que, aún potencialmente, pueda convencer a los inversores internacionales, y la capacidad de gestión que les permita saber proyectarse en los mercados internacionales.

Por ejemplo, en el caso de la farmacéutica humana, la mayoría de la I+D y de los fondos de inversión están puestos en enfermedades oncológicas porque son las que representan el mercado potencial más importante a nivel mundial. Si bien en México estas enfermedades son relevantes, existe otro tipo de enfermedades, como la diabetes, que representa una pandemia nacional, por lo que existe mucho trabajo de investigación a nivel local. Sin embargo, este tipo de enfermedades también afecta gravemente en otros países como en Estados Unidos. ¿Qué se necesita para atraer capital de riesgo que financie la creación de empresas que atiendan este tipo de enfermedades? Fundamentalmente se requiere la elaboración de lineamientos específicos de política pública que ayuden a las empresas en la adquisición de las capacidades antes descritas. Sin una política pública específica para el desarrollo del sector es muy complicado que surjan nuevas empresas nacionales y que las que ya existen logren incorporarse con éxito a los mercados globales.

En términos generales, se puede concluir que las empresas biotecnológicas mexicanas exitosas han optado por estrategias imitativas, lo cual puede ser resultado de la estructura del mercado nacional y de las tendencias hacia la concentración y la conformación de oligopolios a nivel mundial, con una predominancia de las empresas estadounidenses en sectores importantes como el farmacéutico y el agrícola. Sin embargo, la pregunta importante es si este tipo de estrategias alcanzan para desarrollar un sector biotecnológico dinámico que genere productos, procesos y más empresas que compitan con éxito en los mercados internacionales, y por lo mismo genere mayor valor agregado para el país. 


\section{CONCLUSIONES}

Este trabajo planteó como objetivo principal realizar un análisis comparativo de las distintas tecnologías que se patentan en México y en el líder mundial en este sector, Estados Unidos. Asimismo, mediante el uso de las bases de datos de patentes en los dos países se mostró el tipo de tecnologías que desarrollan las empresas mexicanas en ambos mercados.

Los resultados aquí presentados emergen de una propuesta metodológica diseñada para conformar analíticamente al sector biotecnológico, ya que, debido a sus características científicas y tecnológicas de aplicación transversal a diversas áreas industriales, ha sido complejo estructurarlo como un sector tradicional. A partir de la conformación del sector, tomando como base la propuesta de la OCDE, y del uso de las bases de datos del IMPI y del USPTO, se observó que las tecnologías que patentan las empresas mexicanas varía en la conformación de mercados tecnológicos, dependiendo de la región a la que se dirijan (México o Estados Unidos).

En el caso de los registros de las patentes en USPTO las tecnologías denominadas de "aplicación general" dominan la distribución, lo cual demuestra el hecho de que las empresas mexicanas patentan tecnologías con un bajo nivel de especialización, ya que pueden ser usadas en muy diversas áreas industriales. Esto podría entenderse como indicador de su posición tecnológica a nivel internacional y situaría a las empresas nacionales en espacios poco especializados y no enfocados a la generación de productos innovadores, sino en procesos de mayor dominio tecnológico y con poca capacidad de competencia con las empresas transnacionales.

$\mathrm{Al}$ analizar las tecnologías desarrolladas en ambos países a través de las CIPs en USPTO e IMPI, destaca una cierta convergencia tecnológica. Esto significa que en ambas oficinas hay coincidencias de las tecnologías más relevantes para el mercado de la biotecnología. No obstante, un tema relevante es la titularidad de las patentes porque permite identificar la capacidad de producir conocimiento tecnológico por parte de las empresas mexicanas; en este sentido se observa que dicha capacidad es muy baja, ya que en USPTO sólo el $0.06 \%$ del total de las patentes en biotecnología tiene titularidad mexicana y en el caso del IMPI corresponde el $1 \%$, considerando que la participación de centros de investigación y universidades es significativa en estos porcentajes. Lo anterior se conjuga con que los titulares mexicanos reportan una baja participación en las CIPs más importantes, y en conjunto esto expresa una baja capacidad para desarrollar tecnología susceptible de ser valorada en el mercado por parte de empresas mexicanas, además de su incapacidad por situar productos novedosos competitivos a nivel internacional. 
En síntesis, al analizar el patentamiento como estrategia empresarial se constata la participación de algunas pocas empresas mexicanas en este proceso, y se enfatiza el hecho de que como estrategia competitiva es poco usada por el conjunto de empresas. Esto puede deberse a que no cuentan con las capacidades para adquirir conocimiento y desarrollar tecnología, o bien a que el desarrollo innovador del sector se encuentra limitado por el poder oligopólico de grandes empresas transnacionales, que no permiten el desarrollo de capacidades tecnológicas propias.

Dados los requerimientos científicos, tecnológicos y financieros imperantes para el desarrollo de empresas biotecnológicas, además de la estructura de mercado que anima la competencia oligopólica, se establecen dos tipos escenarios en los que las empresas mexicanas pueden participar con diferentes estrategias: el primero es un escenario mundial definido por los mercados internacionales, en el que es muy difícil que las pequeñas y medianas empresas mexicanas compitan con las empresas transnacionales que dominan el mercado. En este escenario la estrategia es situarse en los espacios tecnológicos de menor valor agregado o lo que se ha denominado aquí "tecnologías generales", y tratar de adquirir capacidades tecnológicas y de innovación que les permitan escalar en la elaboración de productos con mayor valor agregado.

El segundo escenario es de carácter local y responde a necesidades de ciertos nichos de mercado o de mercados olvidados por las grandes empresas debido a su valor o tamaño. En este caso la estrategia que han seguido unas pocas empresas mexicanas es desarrollar procesos y/o productos innovadores susceptibles de ser patentados, sobre todo en las oficinas locales, pero con escasa competencia a nivel internacional.

En ambos escenarios las ventanas de oportunidad para las empresas nacionales se sitúan en tecnologías pre-paradigmáticas donde las empresas puedan contar con capacidades o activos complementarios a esas nuevas tecnologías y posibilidades de desarrollar estrategias imitativas tempranas. En estos casos, el carácter emergente de las industrias implica oportunidades de entrada en nichos no explotados por las empresas transnacionales, en subsectores tecnológicos en los que los grandes grupos empresariales del sector aún no estructuran una base de conocimientos coherente e incluso mercados con marcos regulatorios flexibles.

Cabe destacar que los espacios de oportunidades son transitorios, y de allí la importancia de adecuar políticas públicas integrales (de incentivos a la $\mathrm{C}$ y $\mathrm{T}$, de política industrial y de arreglos institucionales) para aquellos momentos en que las oportunidades tecnológicas y la fluidez de la tecnologías sean altas y, por tanto, una mayor incertidumbre permita la coexistencia de estrategias de diversificación de los grandes grupos con la entrada sucesiva de empresas especializadas nuevas que intentan integrar de forma jerárquica o de alianzas distintas etapas de la cadena de valor (Gutman y Lavarello, 2014: 35 y 227-230). 
Una ventaja potencial que debe ser explorada por las propias empresas y los hacedores de política es la gran biodiversidad con que cuentan países como México. Según un estudio realizado por Oldham, Hall y Forero (2013), las patentes registradas en la actualidad se concentran en el $4 \%$ de las especies identificadas. En este sentido, la diversidad biológica existente ofrece aún grandes posibilidades de exploración para aquellos países con gran biodiversidad. Promover estas posibilidades de manera responsable y en estricto apego a los protocolos de $\mathrm{Na}$ goya y la Convención de la Diversidad Biológica es parte del diseño de una política pública específica.

Como ya se ha planteado en otros trabajos del sector (Stezano, 2012; Morales y Amaro 2016), la estrategia de desarrollo y de políticas públicas mexicanas en biotecnología no ha buscado detectar estos espacios y oportunidades para incentivar de manera selectiva a firmas especializadas nacionales con posibilidades de inserción en cadenas globales de valor en estos subsectores tecnológicos. En este sentido, las direcciones y senderos tecnológicos de las empresas nacionales detectadas en el trabajo describen un sector que requiere de políticas de innovación e industriales, lo cual supone apoyo al desarrollo de capacidades (incluyendo el aumento de la infraestructura pública en $\mathrm{C}$ y $\mathrm{T}$ y de la base empresarial biotecnológica nacional) y de intervenciones públicas selectivas sobre la demanda, a fin de desarrollar nuevos perfiles de especialización productiva basados en procesos de rendimientos económicos y especialización crecientes, que favorezcan procesos de cambio estructural en la economía nacional.

\section{REFERENCIAS BIBLIOGRÁFICAS}

Amaro, Marcela; Corona, Juan Manuel y Soria, Manuel (2009), "Incentivos y colaboraciones universidad-empresa: un estudio en el sector biotecnológico mexicano", en Martínez et al. (coords.), Innovación y competitividad en la sociedad del conocimiento, Plaza y Valdés Editores y Concyteg, México, pp. 371-396.

Amaro, Marcela y Morales, Mario Alberto (2016), "Sistema sectorial de innovación biotecnológica en México: análisis y caracterización de sus principales componentes", Revista REDES, vol. 22, núm. 42, pp. 13-40.

(2017), "Vincularse y crecer juntos: iasa y ciba-Tlaxcala", en Villavicencio, Daniel (coord.), Las vicisitudes de la innovación en biotecnología y nanotecnología en México, UAMX-IDRC-Itaca, México, pp. 172-191.

Anandajayasekeram, Ponnia y Gebremedhin, Berhanu (2009), "Integrating innovation systems perspective and value chain analysis in agricultural research for development: Implications and challenges. Improving Productivity and Market 
Success (IPMS) of Ethiopian Farmers Project", Working Paper 16, en: https:// cgspace.cgiar.org/bitstream/handle/10568/513/IPMS\%20Working\%20 paper\%2016.pdf?sequence $=$

Bianchi, Carlos y Torres, Carlos (2018), "Biotecnología para salud humana, estrategias de desarrollo y políticas públicas. Brasil 2004-2016”, en Lavarello, P., Gutman, G. y Sztulwark, S. (eds.), Explorando el camino de la imitación creativa: la industria biofarmacéutica argentina en los 2000, Carolina Kenigstein, Buenos Aires, pp. 60-80.

Carrillo, Graciela y Avendaño, Luisa, (2017), "Nopalimex y la generación de energías limpias”, en Villavicencio, Daniel (coord.), Las vicisitudes de la innovación en biotecnología y nanotecnología en México, UAMX-IDRC-Itaca, México, pp. 192-217.

Castellacci, Fulvio (2007), "Technological regimes and sectoral differences in productivity growth", Industrial and Corporate Change, vol. 16, núm. 6, pp. 1105-1145.

Cimoli, Mario y Dosi, Giovanni (1995), “Technological Paradigms, Patterns of Learning and Development: An Introductory Roadmap", Journal of Evolutionary Economics, vol. 5, núm. 3, pp. 243-268.

Carullo, Juan y Vismara, Felipe (2009), "Inventario de programas de apoyo a proyectos biotecnológicos innovadores", Programa Apoyo al Desarrollo de las Biotecnologías en el Mercosur-Biotech, en: http://docs.biotecsur.org/informes/en/ inventario/11_programas.pdf

Cimoli, Mario y Primi, Annalisa (2008), "Intellectual Property and Development: An Interpretation of the (new) Markets for Knowledge", en Martínez, Jorge (comp.), Knowledge Generation and Protection Intellectual Property, Innovation and Economic Development, Springer, Nueva York, pp. 3-26.

Coriat, Benjamin, Orsi, Fabianne y Weinstein, Oliver (2003), "Does Biotech Reflect a New Science-based Innovation Regime?", Industry and Innovation, vol. 10, núm. 3, pp. 231-253.

De Gortari, Rebeca; Medina, Nelly y Cabrera, Eréndira (2017), "Fungifree Ab ®” en Villavicencio, Daniel (coord.), Las vicisitudes de la innovación en biotecnología y nanotecnología en México, UAMX-IDRC-Itaca, México, pp. 142-171.

Foray, David (2004), The Economics of Knowledge, The MIT Press, London.

Gutman, Graciela y Lavarello, Pablo (2014), Biotecnología industrial en Argentina: estrategias empresariales frente al nuevo paradigma, en: http://www.ceurconicet.gov.ar/imagenes/B.pdf

Jaffe, Adam (1986), "Technological Opportunity and Spillovers of R\&D: Evidence from Firms'Patents, Profits, and Market Value", The American Economic Review, vol. 76, núm. 5, pp. 984-1001.

Klepper, Steven (1997), "Industry Life Cycles", Industrial and Corporate Change, vol. 6 núm. 1, pp. 145-81. 
Franco, Malerba (2004), Sectoral systems of innovation: concepts, issues and analyses of six major sectors in Europe, Cambridge University Press, Cambridge.

Franco, Malerba y Nelson, Richard (2011), "Learning and catching up in different sectoral systems: evidence from six industries", Industrial and Corporate Change, vol. 20, núm. 6, pp. 1645-1675, doi:10.1093/icc/dtr062.

McKelvey, Maureen, Orsenigo, Luigo y Pammolli, Fabio (2004), "Pharmaceuticals analyzed through the lens of a sectoral innovation system", en Franco, Malerba (comp.), Sectoral systems of innovation. Concepts, issues and analyses of six major sector in Europe, Cambridge, Cambridge University Press, pp. 73-119.

Morales, Mario Alberto y Amaro, Marcela Amaro (2017), "Panorama general de la biotecnología en México y el mundo", en Villavicencio, Daniel (coord.), Las vicisitudes de la innovación en biotecnología y nanotecnología en México, UAMX-IDRC-Itaca, México, pp. 33-67.

Morales, Mario Alberto y Villavicencio, Daniel (2015), "Convergencia de capacidades científicas y tecnológicas en el sector de la biotecnología farmacéutica en México", en Morales, Mario Alberto, De Gortary, Rebeca y Stezano, Federico (coords.), Convergencia de conocimiento para beneficio de la sociedad. Tendencias, perspectivas, debates y desafíos, Conacyt- Red Convergencia, México, pp. 139-161.

Nelson, Richard y Winter, Sidney (1977), "Simulation of Schumpeterian Competition”, American Economic Review, vol. 67, núm. 1, pp. 271-276.

OECD (2000), Modern Biotechnology, and Agricultural Markets. A discussion of selected issues. (2009), The Bioeconomy to 2030, Designing a policy agenda, OECD, París. (2015), Bioterchnology update, Newsletter, núm. 29, julio.

Oldham, Paul; Hall, Stephen y Forero, Oscar (2013), "Biological diversity in the patent system", PLoS ONE, vol. 8, núm. 11, pp. 1-16.

Pérez, Carlota (2001), "El cambio tecnológico y las oportunidades de desarrollo como blanco móvil", Revista de la CEPAL, núm. 75, pp. 115-136.

Robert, Verónica; Yoguel Gabriel y Erbes, Analía (2007), "Apropiación y difusión de conocimiento, retornos crecientes", en Stezano, Federico y Velez, G. (eds.), Propuestas interpretativas para una sociedad basada en el conocimiento. Argentina, Colombia, México, Estados Unidos, Canadá, Miño y Dávila, Buenos Aires, pp. 29-65.

Stezano, Federico (2012), "Construcción de redes de transferencia ciencia-industria en el sector de biotecnología en México. Estudio de caso sobre las vinculaciones tecnológicas entre investigadores de Cinvestav Irapuato y Langebio y empresas del sector agro-biotecnológico", Estudios Sociales, vol. 20, núm. 39, pp. 9-38, en: http://www.ciad.mx/archivos/revista-dr/res39/Federico_Stezano.pdf 
Stezano, Federico (2017), "El caso StelaGenomics", en Villavicencio, Daniel (coord.), Las vicisitudes de la innovación en biotecnología y nanotecnología en México, UAMX-IDRC-Itaca, México, pp. 141-170.

Sztulwark, Sebastián (2012), Rentas de innovación en cadenas globales de producción. Provincia de Buenos Aires, Universidad Nacional de General Sarmiento, Argentina.

Trejo, Sergio (2010), Situación de la biotecnología en el mundo, Secretaría de Economía, (SE), Fundación Mexicana para la Innovación y Transferencia de Tecnología en la Pequeña y Mediana Empresa (Funtec, A. C.) y Centro de Investigación en Biotecnología Aplicada, Tlaxcala del Instituto Politécnico Nacional (CIBA-T, IPN), México.

Villavicencio, Daniel y Amaro, Marcela et al. (2014), Yo innovo, él innova, todos innovamos: 15 casos apoyados por el FIT, SE, Conacyt y Cengage Learning, México.

Villavicencio, Daniel y Rivera, Diana (2017), "BioSolutions: emprendimiento y eco-innovación en bioplásticos", en Villavicencio, Daniel (coord.), Las vicisitudes de la innovación en biotecnología y nanotecnología en México, UAMXIDRC-Itaca, México, pp. 243-262.

Wield, David; Chataway, Joanna y Bolo, Maurice (2010), "Issues in the Political Economy of Agricultural Biotechnology", Journal of Agrarian Change, vol. 10, núm. 3, pp. 342-366.

Nota: el presente artículo forma parte de los proyectos papiit IN307116 "Innovación tecnológica, estrategias competitivas y contexto institucional en el sector biotecnológico mexicano" y papiit IA300818 "Procesos sociales en la producción de la ciencia, tecnología y la innovación biotecnológica en México". 